\title{
Strates
}

STRATES Matériaux pour la recherche en sciences sociales

14 | 2008

Espaces du quotidien

\section{Bruce Bégout, La Découverte du quotidien, 2005.}

Jean-Pierre Marchand

\section{(2) OpenEdition}

Journals

Édition électronique

URL : http://journals.openedition.org/strates/6706

DOI : $10.4000 /$ strates. 6706

ISSN : $1777-5442$

Éditeur

Laboratoire Ladyss

Édition imprimée

Date de publication : 1 janvier 2008

Pagination : 275-277

ISBN : 0768-8067

ISSN : 0768-8067

Référence électronique

Jean-Pierre Marchand, «Bruce Bégout, La Découverte du quotidien, 2005. », Strates [En ligne], 14 | 2008, mis en ligne le 04 mars 2013, consulté le 22 septembre 2020. URL : http://journals.openedition.org/ strates/6706; DOI : https://doi.org/10.4000/strates.6706

Ce document a été généré automatiquement le 22 septembre 2020.

Tous droits réservés 


\title{
Bruce Bégout, La Découverte du quotidien, 2005.
}

\author{
Jean-Pierre Marchand
}

\section{RÉFÉRENCE}

Bruce Bégout, La Découverte du quotidien, Paris, Allia, 2005.

1 Découvrir le quotidien, tel est le projet de Bruce Bégout. Il s'agit tout autant d'une découverte au sens où, par exemple, des navigateurs ont un beau jour découvert l'Amérique qu'au sens où cette découverte est l'œuvre d'un dé-couvrir, d'un découvrement: ôter ce qui recouvre, ce qui cache un continent dont le caractère énigmatique est le fait d'un alliage particulièrement résistant d'une proximité enveloppante, créatrice de familiarité et d'une formidable propension à demeurer caché et, partant, profondément méconnu notamment, et comme par définition, par la tradition métaphysique. Pour celle-ci le quotidien est un obstacle aux envols de la pensée dont il faut se libérer. En réalité il est un défi. Sans lui, et telle est une des thèses de l'auteur, aucune œuvre ne serait possible. « Le quotidien, ce qu'il y a de plus difficile à découvrir ", disait Maurice Blanchot que cite en exergue Bruce Bégout.

De manière essentielle la difficulté propre à la découverte du quotidien tient à ce qu'il se présente comme étant nu, non recouvert par quoi ce soit. Découvrir le quotidien c'est alors découvrir ce qui se présente comme n'étant précisément pas à découvrir car tout entier donné dans la croyance en une transparence, en une évidence, en un « aller de soi ». Il est de l'ordre du quotidien de mentir. «Je suis évident, je suis transparent, je vais de soi ", dit le quotidien. Mensonge qui a abusé des philosophes qui ont fait de cette (pseudo) transparence une preuve de médiocrité, de banalité ou d'aliénation. Amateurs d'opacité à vaincre ils n'ont pas vu combien était en réalité opaque, et donc à découvrir, ce qui se présentait faussement mais fonctionnellement comme transparent. Ils étaient dans la méconnaissance même de leur propre quotidienneté, cette « servante » qui leur permettait pourtant de s'envoler à volonté pour le ciel des Idées. 
3 Le projet s'inscrit au reste dans une vision de l'histoire de la philosophie contemporaine où la déconstruction doit cesser de se solidariser de ce qu'elle déconstruit, la métaphysique et ses présupposés, pour décrire l'existence humaine dans sa vérité concrète. Il s'agit « [...] de se coltiner le réel, tout le réel, sans exception ni sélection, le réel gluant et insignifiant, parfois insolite, souvent saugrenu, toujours résistant ». Et c'est un des moments forts de la recherche quand elle se démarque des préjugés qui, par exemple dans Être et temps de Heidegger - et tout en prenant acte de l'esquisse heideggérienne du concept de quotidien - assignent le quotidien au lieu de la déréliction et de la déchéance. Sans esprit de polémique, et en intégrant certains résultats de l'analyse existentiale conduite par Heidegger, l'auteur rompt avec le thème heideggérien de l'historialité comme possibilité essentielle de "nous » sauver du quotidien. La découverte du quotidien est aussi la découverte de ce dont la méconnaissance risque de favoriser l'incitation à se faire le jouet des illusions les plus destructrices du quotidien. Telle serait la nouvelle sagesse - post-moderne diront certains - de cette philosophie non métaphysique : nous faire connaître cette ombre qui nous apporte d'autant plus son aide que nous nous exposons à la lumière de l'inconnu.

4 «Pour l'homme, écrit Bruce Bégout, la vie est [...] par essence - c'est-à-dire par nécessité - quotidienne non pas ontiquement, sur le mode dégradé (et forcément dégradant) de l'esquive et de la médiocrité, de la déchéance et du nivellement, mais ontologiquement, en tant que traduction immédiate du conflit, au sein de l'exister, entre l'extase et la stance ». Ce passage nous introduit au cœur de la problématique. L'approche de la quotidienneté en termes de dégradation, de médiocrité, de déchéance repose sur sa méconnaissance, sur notre adhésion naïve au mensonge du quotidien. Mais que sont « extase » et «stance » dont le conflit est traduit de manière assomptive par la quotidienneté ?

5 L'extase est la constitution même de la conscience humaine en tant qu'elle est toujours « conscience de... ». L'être humain est un être d'expérience, toujours « hors de lui »- en ek-stase - ouvert et exposé au non-moi, à l'inconnu.

6 La stance désigne la nécessité même de vivre, de subsister, d'être " pour soi » et pas seulement, ou pas toujours, « hors de soi ».

7 Or ce à quoi nous expose la structure extatique de la conscience c'est l'Illimité : illimitation de l'espace, du temps, de l'enchaînement des causes. Cette ouverture essentielle à l'Illimité, sans laquelle aucune expérience n'est possible, est cependant source d'inquiétude, et d'une inquiétude telle qu'elle pourrait entraver, perturber voire détruire la possibilité même de vivre. Pour le dire autrement, si nous sommes dans l'inquiétude de l'infini il nous faut faire en sorte que nous puissions nous nourrir tous les jours, nous vêtir, pactiser avec nos rythmes biologiques, etc. Bref la quotidienneté est quotidianisation: domestication jamais terminée de l'Illimité auquel, en tant qu'être d'expérience, nous sommes constitutivement exposés. D'où la nécessité, preuve de sagesse, d'aménager des lieux, des espaces, des temps... Le quotidien est un savoir sans axiomatique. Sans lequel l'Illimité (extase) emporterait notre existence (stance). C'est donc le contraire même de la médiocrité même si, en crise, il peut se faire routine et banalité.

8 La stance n'est en aucun cas une chute de l'extase. Le quotidien est perpétuelle invention car il ne cesse de se nourrir du rapport à l'Illimité. Le quotidien serait semblable à une peau. La peau délimite une micro-région dans un milieu mais ne cesse, 
en même temps, d'échanger avec souplesse avec le milieu. La peau «prouve » le milieu. L'Illimité est comme un milieu transcendantal au sein duquel le quotidien trace des limites qui sont autant de manières d'échanger dans la sécurité avec ce milieu. Le quotidien «prouve » l'Illimité. En ce sens c'est un peu l'habit de la conscience, laquelle est fondamentalement extatique. Ainsi Bruce Bégout ira jusqu'à dire: «De nature dyadique (extase/stance) le quotidien n'est ainsi rien d'autre que l'effort perpétuel d'hospitalité fait à l'étranger. » L'auteur veut dire par là que, face à l'Illimité, on devrait fuir ou se recroqueviller dans une coquille. Au contraire la «peau» du quotidien ne cesse de faire l'hospitalité à l'étranger. Parce qu'il sait répondre en même temps aux exigences de la stance. Il faut lire alors la quatrième partie consacrée aux structures fondamentales du monde quotidien: la maison, le chez soi, les quotidiens privés et publics, le familier, l'habitude, le rire, le rapport à l'étranger, l'hospitalité etc.

9 Mais le livre renouvelle également certaines problématiques. Nous mentionnerons trois des voies que trace un ouvrage lumineux, rigoureux mais non abscons, qui est aussi une programmatique.

10 1. L'analyse de la perception ne peut qu'échouer à nous permettre d'accéder à un donné purement sensible. "[...] le phénomène n'apparaît jamais dans sa simple constitution sensible, rien que phénomène. Il est toujours déjà autre chose que le simple phénomène [...] Ce qui est donné à éprouver immédiatement et en premier lieu dans la vie n'est pas cette simple et pure expérience sensible, mais plutôt une expérience sensée, où les significations pratiques, axiologiques et affectives sont intimement mêlées aux données de la sensibilité pour apparaître instantanément en elles comme choses du monde. »

11 2. Par ailleurs le quotidien et l'histoire ne sont pas en opposition. L'auteur propose une compréhension de l'histoire comme recherche de stratégies de sécurisation induites par la condition humaine en tant qu'elle est invention d'un quotidien. On portera notamment attention aux critiques adressées à Hannah Arendt et à Jan Patocka. Le processus vital, si critiqué par $\mathrm{H}$. Arendt, est en réalité indissociable d'un monde et d'une quotidienneté et n'est donc pas réductible au biologique.

12 3. Dans les derniers chapitres Bruce Bégout, en s'appuyant sur une lecture de textes inédits de Husserl, risque la thèse selon laquelle le quotidien constituerait la fondation ultime de la pensée scientifique.

13 Y-a-t'il alors lieu de penser que la découverte du quotidien constitue un événement majeur? Nous aurions de bonnes raisons de l'espérer tant la pensée du quotidien, pour autant qu'elle soit reconnue, nous maintient en éveil et dans l'accueil des êtres. "Sans l'agitation de l'étrangeté, écrit B. Bégout, le quotidien se défait. » La proposition a sa réciproque : sans pensée du quotidien nous craignons et fuyons l'étrangeté. Et cela n'est jamais le moment. 


\section{AUTEURS}

JEAN-PIERRE MARCHAND

École nationale supérieure

d'architecture de Nancy

2, rue Bastien Lepage,

54001 - Nancy

marchand.fil@wanadoo.fr 